\title{
PERSPECTIVAS DE LA EDUCACIÓN SUPERIOR EN EL CARIBE ANGLÓFONO
}

\author{
Colin Brock*
}

\begin{abstract}
* Cátedra UNESCO "La Educación como una Respuesta Humanitaria" en la Universidad de Oxford, donde ha sido profesor desde 1992. Geógrafo y Educador, fue desde 1977 Director de la Unidad de Educación Internacional en la Universidad de Hull. Ha trabajado en las diversas regiones del mundo en desarrollo para agencias multilaterales y bilaterales, con especial interés en los pequeños estados de las Islas del Caribe, el Pacífico Sur y el Océano Índico. Email: drcbrock@hotmail.com
\end{abstract}

Resumen: En este capítulo, el autor analiza en sus raíces históricas y desarrollo cultural las perspectivas de la Educación Superior en el Caribe anglófono.

Palabras claves: Educación Superior. Caribe anglófono. Economía Política. Cooperación regional e internacional.

Abstract: In this chapter the author analyzes the historical roots and the cultural developments in order to unveil the perspectives of higher education in the Caribbean English speaking countries.

Keywords: Higher Education. Caribbean English speaking countries. Political Economy. Regional and international cooperation.

\section{Introducción}

Ni el término "educación superior" ni "Caribe anglófono" son fáciles de definir. Lo que constituye el sector terciario de un sistema educacional puede variar considerablemente de un país a otro a lo largo del tiempo. Mientras que el término anglófono, en este contexto, puede referirse: a) a la lengua materna de una determinada sociedad; b) al de otra sociedad a la que puede migrar un estudiante en busca de educación superior; c) al medio de instrucción de la educación superior en una sociedad con un lenguaje autóctono diferente o predominante.

Tales complejidades abundan en la región del Caribe, así que debemos comenzar discutiendo una definición de los términos. Desde esta discusión nos iremos hacia una serie de aspectos relativos a la educación superior en el Caribe anglófono que nos llevarán a una serie de conclusiones.

\section{Definición de términos}

El término Caribe se define de varias maneras y a menudo está mal utilizado. Una visión macro, geográfica, es la de la "Cuenca del Caribe", pero como he 
preguntado en otras ocasiones (Brock, 1990), ¿es un concepto regional realista? Para utilizar otro concepto aún más amplio, el Caribe anglófono es parte de la América anglófona, donde en términos del destino de la educación superior de muchas personas del Caribe anglófono es ciertamente importante.

El término "Caribe" está relacionado con el Mar Caribe, siendo ambos nombrados por los amerindios indígenas algunos de los cuales se denominaban "Caribes". Alrededor del Mar Caribe se hallan las tierras del Caribe, algunas de las cuales forman parte de la América Latina, tales como Colombia y Venezuela, mientras que otras forman parte de América anglófona, tales como Belice o Jamaica. El Mar Caribe limita al norte con una cadena de islas relativamente grandes, las Antillas Mayores, cuyas costas limitan al norte con el Golfo de México. Algunas de las tierras que rodean al golfo son predominantemente anglófonas, principalmente los Estados Unidos de América, mientras que otras son de habla hispana, tales como Cuba y México. Las tierras que rodean tanto el golfo como el mar, así como las islas comprendidas en ellos, constituyen la Cuenca del Caribe, que tal como se expresó anteriormente es un concepto a debatir en aquello que no sea en términos físicos y no nos vamos a interesar en ello en lo adelante.

Sin embargo ¡esto se complica! Existen algunos territorios que físicamente están fuera de la Cuenca del Caribe y el Mar Caribe que se podrían denominar "caribeños" por razones culturales e históricas. Como ejemplos tenemos a las Bahamas, las Islas Turcas y Caicos, Barbados, Guyana, Surinam y la Guyana francesa. Aunque esto va más allá del objetivo de este capítulo, se debe notar que, con la excepción de Haití, los demás territorios francófonos de la región, tales como Martinica y Guadalupe, pertenecen políticamente a Francia y en ese sentido ¡no forman parte de la región para nada! De hecho, la falta de un foco "centrífugo" en la región Caribeña se ejemplifica por el hecho de que lo que los geógrafos denominan el "centro" está de hecho en la periferia, esto es, Miami.

El término educación superior también es susceptible de muchas definiciones. Especialmente en lo que se refiere al Caribe anglófono que ya hemos mencionado.

Tradicionalmente, se piensa en la educación superior en términos de universidades, pero es más realista considerarlo en nuestros días como que abarca, si no todo, la mayor parte de la educación formal post-obligatoria. Esto se debe en parte a algunos componentes principales del sistema post-obligatorio que van en otra dirección, tales como la educación vocacional y técnica y la educación y el entrenamiento pedagógicos. También debemos tener en cuenta que en el 
Caribe anglófono, aún después de mucho tiempo de haberse establecido las instituciones post-obligatorias, también están las experiencias de la educación superior de un significativo número de personas que han tenido lugar fuera de la región. Los destinos más importantes han sido anglófonos, Canadá, los Estados Unidos y el Reino Unido. Aunque en su mayoría están más allá de este análisis, si no se toman en consideración, entonces sólo tendremos una comprensión parcial de las opciones y experiencias de la educación superior de los habitantes del Caribe anglófono.

Finalmente, existen unos territorios dentro de esta región donde la educación superior parcial, o predominantemente, es en inglés aunque ésta no sea la lengua materna: por ejemplo, en parte de la Antillas Holandesas y en Puerto Rico.

Errol Miller, uno de los expertos y educadores más prestigiosos del Caribe, resumió el contexto caribeño en la ocasión de un importante seminario regional que tuvo lugar en Trinidad en el 2007:

El Caribe es un addendum del hemisferio occidental.

El Caribe es una subregión distinta pero pequeña y vulnerable, atrapada en la intersección del ejercicio del poder dentro del hemisferio. Estaríamos muy errados si creemos que podemos fiarnos ya sea de la América del Norte o de la América Latina para que se preocupen por nuestro bienestar, a menos que coincida con sus intereses. Así, el Caribe es intrascendente en la política económica del resto del mundo. No está claro hasta que punto los caribeños están plenamente conscientes de esta realidad.

(Miller, 2007, p.14)

Dada las complejidades de la definición analizada anteriormente, las cautas palabras de Miller son bien recibidas. Con esto en mente como telón de fondo, podemos proceder ahora a analizar la educación superior en el Caribe anglófono en relación con una serie de paradigmas contextuales que ayudarán a facilitar una mejor comprensión de la situación contemporánea: consideraciones espaciales y temporales; política económica; factores socio-culturales; cooperación regional e internacional.

Consideraciones espaciales y temporales

"geographie without historie is like unto a carcasse without motion, while historie without geographie wandreth like a vagrant without certaine habitation"

(Anon) 
La región en discusión muestra rasgos geográficos complejos como se indica en las dificultades de la definición citada anteriormente, así como una experiencia histórica muy distinta. Necesitamos comenzar, entonces, examinando la geografía histórica del desarrollo educacional en el Caribe anglófono, que, a diferencia de la mayor parte de la América hispánica, no comienza con una temprana incidencia de las instituciones de educación superior.

Más bien, la combinación de fuerzas del colonialismo y la insularidad ha llevado a una serie de sistemas escolares fragmentados e idiosincrásicos y a un mayor período de dependencia del poder de la metrópoli. Esta, para la mayoría de los territorios que operan actualmente, al menos en parte en inglés, era la Gran Bretaña. La escolarización primaria y secundaria no sólo precede a la terciaria en la experiencia de los individuos, sino también en la historia de la educación en el Caribe anglófono, la mayor parte del cual constituye el "Caribe de la Mancomunidad británica", una entidad neocolonial en sí misma de cierta influencia contemporánea.

El colonialismo implica dependencia, hasta mucho después de haber logrado la independencia política. Así, como cita Brookfield, el estudioso jamaicano Beckford (1972) observó:

El problema más intratable de las sociedades dependientes es la condición colonizada de las mentes de sus gentes.

(Brookfield, 1975, p.161)

La mayor parte de los territorios de lo que se conoce como "Caribe de la Mancomunidad" eran las antiguas colonias de Gran Bretaña. Consecuentemente, ciertas tradiciones y tendencias inherentes en la evolución de la educación formal en la metrópoli se transfirieron a sus colonias. Esto es importante ya que la educación primaria universal no se consiguió en Inglaterra y Gales hasta finales del siglo diecinueve, mientras que la escolarización secundaria jtuvo que esperar la implementación de la Ley de Educación en 1944! Entonces, como en Inglaterra, la escolarización en las colonias caribeñas, siendo la más antigua la de Saint Kitts en 1623, dependía de las misiones cristianas.

El dominio de la iglesia, especialmente de la iglesia anglicana durante los siglos diecisiete y dieciocho, también se extendió a las formas de educación superior que existieron o surgieron. Las universidades renacentistas de Oxford, Cambridge, Dublín, San Andrés, Edimburgo, Glasgow y Aberdeen tuvieron una fuerte influencia en este sentido. Así que no debe sorprendernos que la primera fundación de nivel terciario en el Caribe anglófono sea el Colegio 
Codrington en 1830, un antiguo seminario anglicano en Barbados. Codrington se asoció con la Universidad de Durham en Inglaterra, fundada esta última por la iglesia establecida en 1832, tal como hizo el Colegio Fourah Bay en Sierra Leona, fundado en 1827. La conexión con África Occidental en ese momento es significativa en relación con la emancipación de los esclavos en las Indias Occidentales británicas, y las donaciones del fondo fiduciario Lady Mico apoyando la educación para los esclavos emancipados. Estos y otros apoyos filantrópicos para la misma causa ayudaron a establecer colegios pedagógicos así como escuelas. El primero de ellos, el Colegio Mico en Jamaica, fundado en 1835 fue, de hecho, el primer Colegio Pedagógico en todo el Imperio británico, ¡incluyendo a la madre patria! Sin embargo, en lo que concierne al Caribe anglófono, no existió otro momento cumbre para la educación superior en general. Como plantea Errol Miller, un antiguo director del Colegio Mico:

\begin{abstract}
Entre 1830 cuando el Colegio Codrington se estableció, y 1948 cuando la Universidad de las Indias Occidentales se fundó, habían diez pequeños colegios para formar maestros, cinco colegios más pequeños aún para formar ministros religiosos, unas pocas escuelas de enfermería y dos colegios para entrenar agricultores. Esta magra provisión constituyó la única capacidad de tercer nivel de la subregión
\end{abstract}

(Miller 2007, p. 3).

Se puede ver en este resumen que, a pesar de la emancipación de los esclavos, las necesidades de educación superior que se calcula que existieran eran asumidas, al nivel profesional más alto, por las universidades de la metrópoli, y al nivel profesional más bajo por mujeres - colegios orientados al sector social para formar maestros y enfermeras. Los factores políticos y socio-culturales que subyacían se discutirán en las siguientes secciones de este capítulo, pero primero debemos analizar aspectos del contexto geográfico que tuvieron una influencia en el resumen anterior de Miller; esto es, la dimensión espacial.

Los distintos componentes de la geografía física de esta subregión han sido bosquejados en la definición de términos anterior. Dentro de ello, el Caribe anglófono comprende aquellos territorios que están aún relacionados con Gran Bretaña al extremo de que son miembros de la Mancomunidad Británica, y aquellos que no lo son: casi todo lo que no es la parte sur de los Estados Unidos, especialmente Florida, y dos islas naciones que son de facto colonias de los Estados Unidos -las Islas Vírgenes americanas y Puerto Rico. Las primeras fueron originalmente colonias danesas mientras que la última es de cultura predominantemente española. Esto es, Puerto Rico está tan asociada con la 
migración hacia y desde del noreste de los EE.UU., que sólo es marginalmente del Caribe en contexto. Sin embargo, ofrece oportunidades importantes de educación superior anglófona a las comunidades de las pequeñas islas de la región y es, por tanto, una parte genuina del rompecabezas. Asimismo, el archipiélago de las Islas Vírgenes, una mezcla de los componentes americanos y británicos, ofrece oportunidades muy próximas para aquellos de las Islas Vírgenes británicas (BVIs) que desean acceder a la educación superior. Para la población de las BVIs que son poco más de 10,000 habitantes, no existe la oportunidad de crear una universidad local, aunque existen componentes de la educación terciaria como mencionaremos más adelante. El grado de interrelaciones entre las dos naciones de las Islas Vírgenes se ilustra con el hecho de que mientras las BVIs son todavía una colonia del Reino Unido (RU), es un territorio del dólar americano.

Más allá de estas anómalas situaciones políticas, el resto del Caribe anglófono se compone de la Mancomunidad del Caribe, basada en dos territorios continentales, Belice y Guyana y 15 territorios insulares de las cuales 4 son aún colonias de Gran Bretaña (incluyendo las BVIs). El grado más elevado de insularidad, junto con la pequeñez de escala, condujo a la proliferación de pequeñas instituciones terciarias de orientación vocacional mencionadas por Miller anteriormente. Los aspectos de la pequeñez de escala son intensamente importantes para proveer educación formal a todos los sectores (Bacchus y Brock, 1987). En el contexto de las antiguas "Indias Occidentales británicas", esto se acentuaba por ser ellas "Colonias de la Corona". Esto quería decir que un representante del monarca, no del gobierno, gobernaba a cada una de ellas. Como resultado de ello, cada Gobernador de forma individual ejercía sus propios caprichos y perjuicios idiosincrásicos en términos de oferta educacional. Esto, unido al calidoscopio de misiones cristianas que llegó hasta las más pequeñas comunidades de las islas, conllevó a perfiles altamente diferenciados en la oferta educacional y el desarrollo entre las islas.

¿Qué relación tiene esto con la educación superior? Con la combinación de los legados de cada uno de los Gobernadores - educados en el prestigioso sector privado, entonces Oxbridge -, junto con la gran orientación académica de las misiones, un número importante de jóvenes, especialmente hombres jóvenes, calificados para la enseñanza superior estaban impedidos por las limitaciones de escala y la insularidad. Los que podían obtener el apoyo necesario siguieron a la descendencia de los terratenientes a las universidades metropolitanas. Este elitismo académico llegó a su punto máximo en la 'beca de la isla', adjudicada al de mayores resultados y válida para las universidades de Oxford, Cambridge 
o Londres. Para los que buscaban un título de médico, un lugar en Edimburgo representaba un "brillante premio". Una visión fundamental de la naturaleza de la "educación superior" se creó dentro de estas culturas insulares, que tenía mucho que ver con "la educación para la emigración”. Además, el grado en el que prevaleció en cada una de las islas de las Indias Occidentales británicas variaba considerablemente, en particular con Granada con una dinámica de número de graduados en las profesiones desproporcionada con su pequeño tamaño y población (Brock, 1984). Barbados, pequeña en tamaño pero con una mayor y más integrada población también produce una importante proporción de profesionales, educados y formados en el Reino Unido, los EE.UU. y Canadá, que trabajan en todas las otrora Indias Occidentales británicas. Sin embargo, fiel a la idiosincrasia de estos microestados del Caribe, está la isla de Santa Lucía que ha producido la mayoría de los ganadores del Premio Nobel en el Caribe anglófono.

A pesar de estas anomalías, la combinación de escala, el aislamiento y la dependencia (Brock, 1984) han logrado hacer que la educación superior sea una oportunidad difícil de alcanzar para muchos nativos capaces de haber obtenidos beneficios de ella en términos de su capacidad innata. Esto nos lleva a la cuestión de la economía política y el papel de la educación superior en el desarrollo del Caribe anglófono y, en particular, de la Mancomunidad del Caribe.

\section{Economía Política y la Educación Superior en el Caribe anglófono}

Las universidades siempre han tenido una conexión más fuerte con la economía de lo que se ha reconocido. Es decir, que siempre han tenido una clara función instrumental conectada con las necesidades de sus patrocinadores, los poderes políticos del momento, ya sean religiosos o seglares (Brock, 2007). La evolución de la educación superior en el Caribe anglófono no es la excepción, aunque las decisiones en cuanto a la naturaleza de su desarrollo no han estado necesariamente en armonía con las realidades económicas.

La educación superior en los territorios del Caribe anglófono políticamente relacionados con los EE.UU. está directamente conectada con las oportunidades profesionales en el continente. Los ciudadanos de las Islas Vírgenes de los EE.UU. y Puerto Rico disfrutan de la entrada sin restricciones a todos los 50 estados de la Unión, la mayor economía del mundo. Los ciudadanos de las naciones de la Mancomunidad del Caribe no gozan de entrada sin restricciones, pero un número significativo busca oportunidades para estudiar o 
formarse en los EE.UU. como un conducto para hallar allí empleo calificado y profesional.

A diferencia de la mayoría de las universidades de los EE.UU., las de la Mancomunidad del Caribe se han desarrollado en el contexto del colonialismo y sus secuelas posteriores de dependencia. La Universidad de las Indias Occidentales (UWI), fue fundada como parte de un gesto antes de la independencia por parte del gobierno del Reino Unido que vio homólogos en Nigeria (Universidad de Ibadan), y Uganda (Universidad de Makerere). Al principio eran todos Colegios Universitarios de la Universidad de Londres y servían como un aprendizaje antes de convertirse en instituciones independientes. Este ha sido el mismo proceso que habían pasado también no hacía mucho las universidades provinciales en Inglaterra, tales como Hull, Leicester y Southampton, para así impartir normas y estructuras heredadas a través de los siglos de las antiguas universidades. En el caso de la UWI, San Andrés también participó. Hubo pocos esfuerzos para idear un nuevo tipo de universidad donde algunos de sus programas, al menos, estuvieran referidos a los desafíos económicos y políticos de la subregión. El Colegio Universitario era considerado, sin embargo, como una institución regional fundada en 1948 en un momento en que todos los territorios eran todavía colonias y por lo tanto parte de un todo teórico, las Indias Occidentales Británicas. Esta situación pasó de la teoría a la realidad con la concesión de la independencia política conjunta a todas estas colonias bajo la forma de la nefasta Federación de las Indias Occidentales en 1958.

Desde el principio la UWI se concibió con tres campus, en Jamaica, Trinidad y Barbados. Los dos primeros fueron ubicados en las islas más pobladas, mientras que Barbados fue visto como el campus de las pequeñas islas de Barlovento y Sotavento. Cada campus ofrecía disciplinas básicas tales como matemáticas, inglés e historia, mientras que los campus aplicados se concentraban en un mismo lugar, como la medicina en Jamaica y la agricultura en Trinidad. De hecho, el campus de San Agustín en Trinidad había sido el antiguo Colegio Imperial de Agricultura que servía a todas las colonias del Imperio.

El papel regional de la Universidad de las Indias Occidentales fue sometido a una dura prueba con la desintegración de la Federación después de sólo cuatro años en 1962. Los cuatro componentes más poderosos, Jamaica, Barbados, Trinidad y Tobago y Guyana se convirtieron en naciones independientes, mientras que el resto cambiaron de forma, ya sea en un nuevo Estado Asociado con el Reino Unido o volvieron a ser colonias de nuevo. Nunca ha existido una economía regional como tal, sólo lo que Altbach y Kelly (1978) denominan "colonialismo clásico" que, por definición, vincula a las economías de todos los 
componentes a la del Reino Unido. A partir de 1962, hubo cuatro economías políticas totalmente independientes y una serie de las más pequeñas todavía atadas de diferentes maneras y en diversos grados a la metrópoli y desde 1973 a la Comunidad Económica Europea. Guyana fundó su propia universidad en 1963, mientras que los tres campus activos de la UWI inevitablemente se relacionaron más estrechamente con Jamaica, Trinidad y Barbados, respectivamente, a la vez que eran parte todavía de una universidad regional.

El colonialismo clásico dio paso al colonialismo regional, donde el pez más grande del estanque, sobre todo Jamaica, se convirtió en líder regional en lugar de ser un componente. Esto se reflejó en la UWI cuando Roy Marshall, un antiguo estudioso de la isla de Barbados y profesor de derecho en la Universidad de Sheffield fue nombrado Rector. Al ser el primer no-jamaiquino en ocupar ese puesto, y ser visto como un "pequeño isleño", fue sometido a un boicot y se trasladó a otro puesto después de sólo unos años.

Con la diversificación de sus economías independientes, las tres naciones campus de la UWI ofrecían unas perspectivas de empleo más atractivas a los graduados que habían venido de las islas más pequeñas con sus concentradas economías. Jamaica, con más de dos millones de personas tenía una cierta masa crítica, la cercanía a los EE.UU. y, bajo Michel Manley, una asociación con la cercana Cuba post-revolucionaria. En Trinidad y Tobago había riqueza petrolera, mientras que Barbados actuaba como un almacén de materiales con valor añadido para los productos manufacturados, no sólo para casa sino también para las islas de Barlovento y Sotavento.

La diversificación de estas tres economías tradicionales también convirtió el currículo académico tradicional de las escuelas en otros más importantes que estaban directamente relacionados con las necesidades de los empleadores locales. En otras palabras, había una correspondencia positiva entre el plan de estudios y la economía, con una progresión a la educación superior en el campus local que fuera tanto geográficamente conveniente como pertinente desde el punto de vista ocupacional. Los estudiantes de las islas más pequeñas eran atraídos por el empleo en su isla del campus después de la graduación, de ahí la noción de colonialismo regional. La fuga de cerebros de colonia a la metrópoli fue sustituida por una versión más local que era un poco discordante con la teoría de una universidad regional.

Para las islas más pequeñas, microestados y mini-estados, siempre había habido un problema de ofrecer un programa de estudios, que fuera importante al mismo tiempo, para las necesidades del pequeño sector moderno de sus economías y la economía local básica y dominante de la mayoría, a menudo muy 
vulnerable (Brock y Smawfield, 1988). Como un intento de servir a los pequeños países insulares, la UWI ha establecido pequeñas facilidades extra-muros en las instalaciones de cada uno de ellos. A continuación, coordinó dicha prestación estableciendo un Centro de Estudios de Programas en el Extranjero en el campus de Cave Hill en Barbados. Si bien se trataba de un verdadero esfuerzo fue, durante algún tiempo, incapaz de superar las graves limitaciones de escala en las islas menores, en especial en los microestados tales como las Islas Vírgenes Británicas. Aunque estas limitaciones eran importantes, también un persistente colonialismo regional esperaba que los estudiantes acudieran a los principales campus y realizaran los tradicionales cursos de tiempo completo. Al mismo tiempo, los Estados Asociados, un poco más grandes y políticamente más fuertes comenzaron a pensar en términos del desarrollo de una capacidad limitada terciaria sobre la base de sus actuales Colegios pedagógicos y técnicos. Estas iniciativas tenían el doble objetivo de responder a las necesidades locales para fortalecer la capacitación y proporcionar una base para realizar la licenciatura en la Universidad de las Indias Occidentales. El Colegio de las Bahamas fue uno de los primeros modelos, y el otro ejemplo más notable es probablemente el Colegio Sir Arthur Lewis en Santa Lucía. Este último fue denominado así por uno de los tres ganadores del Premio Nobel de la isla-, y desarrollado sobre las masivas fortificaciones de Morne Fortune encima de la capital, Castries. Además del Colegio Pedagógico y el Colegio Tecnológico, había un Colegio Técnico Pedagógico, un centro de planificación urbana de Naciones Unidas, un centro de servicios de la escuela, y cerca el Centro Rockefeller para la Investigación de la Bilharzia. Este grupo, aunque no unificado, generó una masa crítica de profesionales de alto nivel que, aunque informalmente, ofrecieron al complejo y a la isla una cierta visibilidad en la enseñanza superior (1995)

Harvey y Marrett, (2008) estiman que en la actualidad hay "cerca de 30 universidades no-hispano parlantes". Y luego afirman:

Desde el 2002 en la región se ha visto una tendencia a la creación (real o prevista) de otras universidades, tanto públicas como privadas, en gran parte a través de la fusión de las actuales instituciones de enseñanza superior. Otra tendencia de los últimos 20 o más años es el establecimiento de universidades en el extranjero en ciertos países caribeños, algunos de los cuales son accesibles a los ciudadanos del Caribe (p. 301).

Cuántas de estas nuevas instituciones realmente califican bajo las normas internacionales para el título de "universidad", es lo que habría que ver. 
Harvey y Marrett (2008) también citan la revisión de Roberts (2004), que examina el desempeño de 15 países del CARICOM (Comunidad del Caribe) respecto a la matrícula terciaria desde los 17 a los 24 años de edad. CARICOM ha establecido una meta de que el $15 \%$ de ese grupo etáreo debe estar matriculado en la educación terciaria para el año 2005. Los que superan esta meta son Barbados (29\%), las Bahamas (21), Islas Vírgenes Británicas (20), Islas Turcas y Caicos (20), Granada (18), St Kitts / Nevis (16) y Antigua (16). Trinidad y Tobago (15) apenas alcanzó a pesar de que, como Barbados, tiene un campus de la UWI. Luego siguieron Dominica (14), San Vicente y las Granadinas (12), Guyana (9), Santa Lucía (8), Islas Caimán (8), Montserrat (6), y Anguila (4).

Hay muchas idiosincrasias detrás de estas cifras, incluso asumiendo que sean exactas. El pequeño microestado de las Islas Turcas y Caicos podría beneficiarse por su proximidad a las Bahamas y los EE.UU. continental; las Islas Vírgenes Británicas, otro minúsculo microestado, experimentaron el ascenso de un número importante de sus profesores a través de un programa de cinco años con la Universidad de Hull, Inglaterra - dirigida por el autor - a partir de 1984 -1989; Granada acoge ahora la gran Universidad de San Jorge, una "institución en el extranjero" de los EE.UU. Por el lado relativamente bajo está Guyana, que a pesar de tener su propia universidad es relativamente grande territorialmente y económicamente pobre, y Trinidad, que, a pesar de contar con un campus de la UWI, tiene una población relativamente grande.

Aunque los porcentajes de matrícula en la educación superior en el grupo etáreo de 17-24 años van en aumento, se trata de un proceso lento, y se mantendrán muy por debajo del de los países desarrollados de América del Norte y Europa, que van del 40 al 80\%. Jamaica, el tercero y principal país-campus de la UWI no aparece en las cifras del CARICOM, pero con más de dos millones de personas está en otra liga demográfica. Junto a los principales campus de la UWI está la Universidad Tecnológica de Jamaica, fundada en 1998, centrada en las cuestiones nacionales y locales relacionadas con el desarrollo económico y social, y que también está aumentando la capacidad para la matriculación en la enseñanza superior. Jamaica tiene una economía relativamente diversificada, en comparación con las islas más pequeñas, miembros del CARICOM. No obstante, al menos el desarrollo de una instalación de Colegios Universitarios en muchos de los microestados tiene la capacidad de proporcionar enseñanza del primer ciclo de los estudios de grado para titulaciones en la Universidad de las Indias Occidentales. Esto tiene el efecto de retrasar el éxodo académico, así como la conexión, aunque limitada, con las necesidades de diversificación del empleo de las pequeñas economías insulares. 
A pesar de estos avances, todavía existen considerables presiones y desafíos que enfrenta la educación superior en el Caribe anglófono. Los gobiernos involucrados están exigiendo más de los sistemas de educación superior, en términos de acceso, equidad y calidad, al tiempo que les ofrecen menor apoyo financiero. (Howe, 2005).

Es evidente que se necesitan más fondos, pero el los que dispone el sector privado, pueden traer consigo una dilución de las normas de calidad como ha ocurrido en muchas partes del mundo en desarrollo, incluida la cercana América Latina.

Las instituciones privadas, a menos que sean filantrópicas, es poco probable que ofrezcan oportunidades a las mayorías pobres de la población. En consecuencia, el talento endémico que se encuentra latente en todas las sociedades, y que necesita desesperadamente el Caribe de habla inglesa, se mantendrá sin explotar con esta modalidad de ES.

\section{Factores Socioculturales.}

\section{Educación y clase social: una herencia disfuncional de la educación colonial.}

En esta discusión habría que tener en cuenta factores sociales y culturales, matizados y coloreados fuertemente por la experiencia colonial. Esto implica que la educación formal es ofrecida con una determinada visión política, pero luego resulta culturalmente integrada a la matriz autóctona.

En lo que concierne a la enseñanza superior en particular, el largo período de dominio colonial experimentado por las naciones del Caribe Anglófono contemporáneo, incluyó la implantación de los modelos europeos de universidad. Muchos de los territorios experimentaron el dominio de una sucesión de potencias coloniales, pero el modelo europeo originario del renacimiento predominó a nivel mundial y en el Caribe. La variante inglesa de este modelo de universidad, sin embargo, se caracterizó por un elitismo más agudo, conectado con el dominio del sector privado en la educación secundaria.

Los hijos de la plantocracia (aristocracia terrateniente) fueron enviados a dichas escuelas, que fueron modelos de escuelas secundarias sumamente selectivas, a menudo con el carácter de fundaciones religiosas. Al igual que en Inglaterra, la educación estaba estrechamente vinculada a la clase social. Esto se encuentra muy bien ilustrado en la famosa serie de libros de sociología "Perspectivas de las Indias Occidentales", editado por Lambros Comitas y 
David Lowenthal, a principios de los años 1970, en especial bajo el título de "Consecuencias de la Clase y el Color" (1973). Eric Williams, primer ministro de Trinidad y Tobago y autor del capítulo sobre "Educación en las Indias Occidentales Británicas", comentó con relación a este tipo de escuela secundaria:

Al igual que en el nivel primario, el plan de estudios es divorciado de las verdaderas necesidades de la comunidad y de la actividad y de la experiencia de los alumnos. Se podría decir calificadamente que la escuela secundaria prácticamente hace un fetiche de esta irrealidad. La enseñanza secundaria es tan severamente restringida a pocos, que la educación inglesa que esta proporciona se convierte en un signo de distinción social (Williams, 1973, p. 151).

En una referencia más explícita a la clase social, y a la clase media en particular, C.L.R James, una figura clave de las ciencias sociales en Trinidad, prologa su capítulo así: Nuestras clases medias, no preparadas, y que han recibido una educación inadecuada, deben enfrentar repentinamente los enormes líos dejados por los imperialistas (1973, p 79). ¿Con la UWI en su primera infancia en el momento de la escribir este libro, podría ser que la naturaleza de dicha institución era uno 'de los líos' en cuestión? Es interesante, y quizás saludable, la observación que en las 1510 páginas de este cuarteto espléndido de libros, no exista ninguna sección, ni siquiera un párrafo, sobre la UWI. Un resumen editorial del capítulo por C.L.R. James lo pone así:

C.L.R. James se refiere aquí a la Clase Media del Caribe Anglófono como mal preparada para el liderazgo. Excluida de la arena productiva por la solidaridad de la elite y su propio concepto de cortesía, carece de experiencia en la dirección de los asuntos económicos. Entrenada en una visión jerárquica de sociedad, la clase media antillana, en la opinión de James, debe reeducarse para trabajar para el más amplio interés nacional (ibíd. p 78).

Estos autores, pertenecientes ellos mismos al Caribe Anglófono, hablan de una clase de gente, cuyos líderes se han educado en la UIW o en una institución metropolitana o norteamericana de la misma índole. Entonces una pregunta fundamental surge. ¿Las instituciones de enseñanza superior del Caribe Anglófono, y sobre todo la UWI, tienen lo necesario para proveer el tipo de liderazgo educativo que hace falta en un escenario regional de mini-estados fragmentados, o bien en el amplio escenario internacional globalizado del que forman parte? 
Estos son temas a los que nos referiremos en la sección penúltima de este capítulo sobre la cooperación regional e internacional, pero antes de esto tenemos que considerar otro aspecto de importancia sociocultural que marca su impronta en el desarrollo de la ES en el Caribe Anglófono: el de género.

\section{Género y educación en el contexto del Caribe Anglófono.}

Hay una literatura considerable sobre el género y la educación en países en vía de desarrollo, sobre todo referido a las desventajas femeninas. El autor ha contribuido a ello (Brock y Cammish, 1997). La Región caribeña, y sobre todo las sociedades de las islas del Caribe Anglófono, no se ajustan a las tendencias mayoritarias de estos países en lo que se refiere a educación y género. Debido a esto fue que incluimos Jamaica entre los siete países en nuestro estudio. Esta cuestión podría ser de importancia para la enseñanza superior en el Caribe Anglófono, pues el equilibrio de género en la educación primaria y secundaria bien podría tener implicaciones para los componentes del sector post-obligatorio.

Jamaica es un país adecuado para comenzar una breve exposición del tema: es la tierra de Edith Clarke, el título de cuyo trabajo seminal "Mi Madre que fue MI Padre" (1957) lo dice todo. Una sucesión de escritores como Lowenthal (1972), y Smith (1965), han ilustrado luego el modelo de ventaja femenina en educación en todas partes de la sub-región, en lo que concierne a los mayoritarios estratos más pobres de las poblaciones de las Islas. La esencia de la cuestión radica en la estructura matrifocal de la mayoría de las sociedades del Caribe Anglófono.

Es decir, debido a una herencia de desequilibrio sexual en las poblaciones esclavas previo a la emancipación, se desarrolló un modelo de estructura familiar, por el cual la madre tenía en la familia el control de la toma de decisiones por omisión. Los hombres adultos engendrarían la descendencia mediante una sucesión de compañeros, de modo que las mujeres adultas también experimentaron tal sucesión, conocida por los antropólogos como la monogamia sucesiva. Sin embargo, a diferencia de sociedades matriarcales genuinas, las madres antillanas no heredaron el poder o la riqueza, pero sí tuvieron el poder de tomar de decisiones. Esto se extendió a las cuestiones de educación, y una de las ventajas de estas pequeñas comunidades isleñas, fue que era relativamente fácil en los días coloniales, y desde entonces, el acceso no solamente masculino, sino también femenino, a la enseñanza primaria universal. En la generalidad del "Tercer Mundo", la mayor parte de las sociedades son patriarcales, y las 
decisiones acerca de si una mujer asiste o no a la escuela son hechas por el padre, que no está siempre presente, pero que es más poderoso que su compañera. Esto es en parte debido a un nivel superior de educación y a la tendencia para la mayor parte de mujeres de origen campesino de seguir la jerarquía sexual de edad (Todd, 1987).

Las mujeres antillanas - en su mayoría pertenecientes a los estratos más pobres- con frecuencia ejercían su poder incontestado de decisión a favor de sus hijas. Los muchachos pobres podían sobrevivir mediante un cierto tipo de comercio ambulatorio de poca magnitud que se practica en las calles del Caribe Anglófono. Una vez en la escuela, las hembras fueron capaces de demostrar sus actitudes más positivas para estudiar y progresaron más rápido que los varones. En el contexto del Caribe Anglófono, la selección para escuelas secundarias, a menudo conocida como " la Entrada Común", significó que más muchachas tuvieron éxito en el ingreso - y en los estudios - que los muchachos. Con el advenimiento de la UWI y otras oportunidades de estudios terciarios, las muchachas también dominaron, de modo que ahora la mayoría de estudiantes en la universidad es femenina en una proporción de 2:1. Sin embargo, según Howe (2005, p. 59):

La atención tiene que ser enfocada sobre la baja tasa de matrícula de mujeres en Ingeniería y facultades de ciencia, y en si las mujeres de los grupos socioeconómicos más bajos y desheredados, realmente tienen acceso a la educación terciaria en números equitativos. Sin embargo, la preocupación mayor debe ser expresada acerca de la baja tasa de matrícula de los hombres y su pobre desempeño en todos los sectores educativos.

De ahí la importancia para la ES. Sin embargo, se debe decir que las clases medias ya mencionadas adoptaron una estructura familiar más estable, debido en parte a sus ingresos superiores. Esto implicó que brindasen un apoyo similar a sus hijos varones y hembras con relación a las oportunidades de educación. Por eso, a pesar de la ventaja femenina en la mayoría, y las oportunidades para muchachas de clase media, las desventajas de género han prevalecido, aún en áreas donde el poder y la riqueza predominan. Esto es especialmente así en el terreno de la política donde, según C.L.R James y Eric Williams, las capacidades de liderazgo parecen haber estado ausentes en el pasado en la subregión. Hubo algunas excepciones, pues tanto Dominica como Jamaica han tenido mujeres como primeros ministros. ¿Será quizás en el Caribe Anglófono que la tesis reciente de Desmond Morris (2008), de que el mundo sería un mejor lugar de ser controlado por mujeres, será puesta a prueba? 
Según Errol Miller (1986) en su polémico libro "La marginalización del varón negro: ideas sobre el desarrollo de la profesión de maestro", uno de los aspectos del avance de las hembras a expensas de los varones, ha sido la feminización de la profesión de maestro. Esto, según él, entre otros aspectos, tuvo un efecto negativo sobre la ya desventajosa situación de los varones en las escuelas. La tesis fue impugnada con ferocidad. Una vez más, el Caribe está en desacuerdo con la mayoría del mundo en desarrollo en cuanto a esto. En cualquier otro lugar, en países en vía de desarrollo, debido a las ventajas masculinas, la mayor parte de los profesores son hombres, aún en el nivel primario. La situación en el Caribe Anglófono es significativa para la enseñanza superior. Hasta mediados del siglo XX, la proliferación de pequeñas universidades de formación de profesores representó el sector terciario. Ahora, los antiguos colegios de profesores forman el corazón de los colegios de la comunidad ya mencionados, que han hecho tanto para hacer avanzar en oportunidades postobligatorias de educación a varones y hembras igualmente. Ellos también han sido capaces de fomentar el desarrollo de las habilidades necesarias para las economías de las diversas islas, y proporcionan una vía para obtener los primeros grados en la UWI.

\section{Cooperación Regional e Internacional}

Durante seis décadas, la UWI ha sido la primera institución de enseñanza superior en el Caribe Anglófono. La discusión a que nos hemos referido, de la variedad de influencias contextuales en medio de las cuáles la ES ha funcionado, ha tendido a sugerir que no pueda ser el ideal para afrontar los desafíos que enfrenta la ES en un mundo globalizado al inicio de un nuevo milenio. La sensible ubicación geopolítica entre América del Norte y América Latina, la fragmentación de la geografía política del territorio, y la vulnerabilidad económica relativa de la mayoría de estas pequeñas naciones, dan lugar a un escenario difícil. Con una situación logística tan problemática, y llamadas ocasionales a los tres recintos universitarios de la UWI para que se conviertan en universidades independientes, ella sobrevive y mejora dado que posee al menos dos fuerzas fundamentales. En primer lugar dado que se creó con un carácter de institución regional con una estructura regional. En segundo lugar, a pesar de que algunos la perciben como parte de una tradición elitista, sin duda es una institución de elevado nivel académico. La UWI tiene una calidad adecuada para enfrentar los desafíos contemporáneos. 
La UWI ha sido apoyada en su papel regional y en su identidad, por el paralelo Consejo de Exámenes Caribeño (CXC). Esta institución proporciona a la mayoría de las naciones del Caribe Anglófono, asesoría en planes de estudios y evaluación a nivel secundario de probada calidad académica y profesional. Aunque basado en el modelo británico de evaluación independiente pública, más bien que en el modelo de EE.UU. basado en la escuela, este modelo sabiamente se ha alejado de la estrechez de la variante inglesa y se parece más a la más amplia estructura de formación de los planes de estudios escoceses y europeos. En ámbitos relevantes, como estudios sociales y ciencias naturales, CXC ha tenido logros importantes en temas regionales. Todo esto ha apoyado la evolución de la UWI como una universidad regional que mantiene altas normas académicas de calidad.

Esta calidad es importante, en el contexto de la preocupación contemporánea internacional por la garantía de la calidad en la ES a escala global. Desde 1998, la UNESCO ha liderado el camino, a través de una serie de Foros Globales e iniciativas regionales, en una tentativa de combatir la dilución potencial de normas académicas en el nivel universitario, debido a la proliferación de programas en línea y de universidades privadas de dudosa calidad.

El Caribe ha tenido muestras de ambos, aunque principalmente en los territorios del Caribe hispánico como República Dominicana. Miller (2007) ha descrito como los gobiernos nacionales en el Caribe Anglófono han contenido, al menos en cierto grado, la expansión de la educación terciaria privada.

Mientras los Gobiernos seguían una política de financiar una sola universidad regional y una variedad de colegios nacionales financiados también del tesoro público, una nueva dimensión fue añadida al paisaje cuando el parlamento de Granada, en julio de 1976, aprobó la Ley que autorizó a la Escuela Universitaria de Medicina Saint George a abrir sus puertas en enero de 1977. Desde entonces otras instituciones similares de carácter privado, denominadas "universidades en el exterior" (off-shore universities) han sido establecidas en Belice, en Saint Kitts y Nevis y en otros países de la región (Miller, 2007 pp. 3-4). .

Él aplaude el hecho de que las Leyes del Parlamento han validado su estatus como universidades, y afirma que estas instituciones son una parte del paisaje de la enseñanza superior en el Caribe Anglófono. Con la UWI como una especie de "patrón oro" de lo que debe ser un nivel adecuado en la visión de los gobiernos de las Islas, esto desempeña un papel clave en el tipo de comparaciones (benchmarking) que la UNESCO desea promover. 
¿Pueden la UWI y otras instituciones de enseñanza superior de prestigio en el Caribe Anglófono jugar su papel en lo que Altbach y Peterson (2007) ven como "'Desafíos Globales e Ideas Innovadoras" exigidas a la Enseñanza superior en el Nuevo Siglo? En su introducción bajo el título de Realidades Subyacentes de la Enseñanza superior en el siglo XXI, Altbach identifica los problemas clave siguientes: masificación; el interfaz entre bien público y privado (mercantilización); la economía basada en el conocimiento; y la tecnología de la información.

Temas similares fueron identificados en la publicación del Banco mundial, Construir Sociedades de Conocimiento: Nuevos Retos para la Educación Terciaria (2002), en la cual se considera un problema clave la contribución de la educación terciaria al desarrollo económico y social. Esto, tradicionalmente, no ha sido visto como una misión de las universidades, excepto en ciertas áreas de investigación, y la UWI no es ninguna excepción al respecto. Se requiere un cambio radical de cultura en la educación terciaria y este debe producirse. La cuestión de la masificación de la enseñanza superior en el Caribe Anglófono, presenta rasgos diferentes a la mayoría de los sistemas de ES del mundo en desarrollo. En general, la región tiene un perfil demográfico envejecido, semejante al de Gran Bretaña. Esto quiere decir que el imperativo masificación de la ES, debe ser dirigido a estudiantes de edad madura y a los estudiantes más jóvenes. En sociedades urbanas densamente pobladas, esto podría ser alcanzado a través de estudios de media jornada, en instituciones fácilmente accesibles; pero en un escenario de pequeños estados insulares diseminados, esto sólo parece factible hasta el nivel de colegios universitarios de la comunidad.

Programas virtuales de nivel de grado, podrían solucionar este problema, que nos trae a otro de los imperativos de Altbach, la tecnología de la información. La UWI tiene que mejorar su capacidad y ajustar sus programas en cuanto a esto. La creciente interacción entre un número de universidades privadas en aumento y el "patrón oro" de sector público establecido, también sería provechosa. Esto levantaría el estándar de las instituciones privadas legítimas y ayudaría a eliminar las fábricas de títulos.

La UWI como "”el patrón oro" y la única institución realmente regional de enseñanza superior, necesitará el apoyo de otros marcos regionales, sobre todo del CSME, diseñado para combatir las limitantes a escala nacional. El CSME no es completamente anglófono, además de las 12 naciones independientes de habla inglesa que lo integran, Surinam y Haití son también miembros. Como Miller (2007) nos recuerda, estas catorce naciones son los únicos regimenes en el Caribe que no son, ni actual ni potencialmente: 
"parte de ningún bloque continental poderoso - o potencialmente poderoso - de países... y están por ello obligados a hacer causa común en la construcción de su futuro"

Curiosamente, la relativamente gran escala de Haití en términos de población, y su candidatura para ser el país más pobre en el mundo, presenta un tipo totalmente diferente de nivel de desafío a la enseñanza superior en el Caribe Anglófono, en su papel en el siglo veintiuno de contribuir al desarrollo económico y social. El Caribe está lleno de sorpresas, y esto incluye a la educación superior.

\section{Bibliografía y Referencias}

Altbach, P.G. and Peterson, P.M. (2007), (Eds), Higher Education in the New Century: Global Challenges and Innovative Markets, Rotterdam, Boston College and UNESCO.

Altbach, P.G. and Kelly, G.P. (1978), (Eds), Education and Colonialism, New York, Longman.

Austin, I. and Marriott, C. (Eds), (2002), Adult Education in Caribbean Universities,

Kingston, UNESCO

Bacchus, M.K. and Brock, C. (1987) (Eds), The Challenge of Scale: Educational Development for the Small States of the Commonwealth, London, Commonwealth Secretariat.

Beckford, G.L. (1972) Persistent Poverty: underdevelopment in plantation economies of The Third World, Oxford, Oxford University Press.

Brock, C. (2007) 'Historical and Societal Roots of Regulation and Accreditation for Higher Education for Quality Assurance, in: GUNI, Higher Education in the World 2007: Accreditation for Quality Assurance: What is at Stake? Basingstoke, Palgrave Macmillan, pp24-36.

Brock, C. and Cammish, N.K. (1997), Factors Affecting Female Participation in Education in Seven Developing Countries, London, DfID. 
Brock, C. (1990), 'The Caribbean Basin - A Realistic regional Concept'? in: Brock, C. and Clarkson, D. Education in Central America and the Caribbean, London and New York, Routledge, pp 1-6.

Brock, C. and Smawfield, D. (1988) 'Education and Development: the Issue of Small States', in: Educational Review, 40:10, pp 227-239.

Brock, C. (1988) 'Beyond the Fringe? Small States and the Provision of Education', in:Comparative Education , 24:2, pp167-179.

Brock, C. (1984), Scale, Isolation and Development: Aspects of Education in Island Developing and Other Specially Disadvantaged States of the Commonwealth, London, Commonwealth Secretariat.

Brock, C. (1984), 'Education and the Multicultural Caribbean', in: Corner, T. (Ed), Education in Multicultural Societies, London, Croom Helm, pp 156196.

Brookfield,H.C. (1975) Interdependent Development, London, Methuen.

Figueroa, J. J. (1971), Society, Schools and progress in the West Indies, Oxford, Pergamon.

Harvey, C. and Marrett, C. (2008) 'Higher Education for Human and Social Development in the Caribbean', in: GUNI, Higher Education: New Challenges and Emerging Roles for Human and Social Development, Basingstoke, GUNI/ Palgrave Macmillan pp 300-302.

Howe, G.D. (2005), 'Contending with Change:Reviewing Tertiary Education in the English Speaking Caribbean, in: http.//iesalc.unesco.org.ve/

Jennings, K. (2001), 'Teacher Education in Selected Countries in the Commonwealth Caribbean: the Ideal of Policy versus the Reality of Practice, in: Comparative Education, 37:1, pp 107-134.

Lopez Segrera, F. (2006b) "Higher Education in the Caribbean". In GUNI, Higher Education in the World 2006. The financing of universities. Palgrave Macmillan, Basingstoke.

Louisy, C.P. (1995), 'Tertiary Education in St Lucia: Implications for Small Island States', in : International Journal of Educational Development, 15:2, p196 (PhD Abstract, University of Bristol).

Lowenthal, D. (1972), West Indian Societies, New York, Oxford University Press. 
Lowenthal, D. and Comitas, L. (Eds) (1973) West Indian Perspectives: Consequences of Class and Colour, New York, Anchor Books.

James, C.R.L. (1973), 'The Middle Classes', in Lowenthal, D. and Comitas, L. (op cit), pp 79-94.

Miller, E. (2007), Keynote Address to a Seminar on : Research and Higher Education Policies for Transforming Societies: Perspectives from Latin America and the Caribbean, Trinidad, July $19^{\text {th }}$.

Miller, E. (1986), Marginalisation of the Black Male: insights from the development of the teaching profession

Morris, D. (2008), The Naked Man: A Study of the Male Body, London, Jonathan Cape.

Roberts, V. (2004) Widening Access to Tertiary Education: Revisiting the Fifteen Percent Target (unpublished document)

Smith, M.G. (1965) The Plural Society in the British West Indies, Berkeley, University of California Press.

Todd, E. (1987), The Causes of Progress: Culture, Authority and Change , Oxford, Basil Blackwell.

Williams, E. (1973) 'Education in the British West Indies' in: Lowenthal, D. and Comitas, L. (op cit), pp 148-168.

World Bank (2002), Constructing Knowledge Societies: New Challenges for Tertiary Education, Washington DC, The World Bank. 